\title{
Respiratory trigger signal generation by means of a stretchable sensor array
}

Eugen Koch, Andreas Dietzel, TU Braunschweig Institute of Microtechnology, Alte Salzdahlumer Str. 203, 38124 Braunschweig, Deutschland, eugen.koch@tu-bs.de

\begin{abstract}
Respiratory monitoring is a clinical method which helps to examine the medical condition of patients. Patients diagnosed with types of respiratory distress are often supported through artificial respiration. To be able to adapt and synchronize airway pressures and flows to the patient's own breathing for improved respiration efficiency, intelligent sensors are needed to detect the beginning and ending of the breathing cycle. An ultrathin and stretchable $6 \times 6$ sensor array with skin-like properties is presented that is used to generate a trigger signal which is suitable to control and synchronize artificial respiration with the patient's own breathing. Stretchability of the sensor array is achieved by fs-laser structuring of the thin polyimide sensor substrate resulting in small sensor islands connected via slender meandering electrical leads. The resulting stretchable sensor grid is embedded in layers of PDMS whereby a skin-friendly sensor patch is created. To simulate respiration an externally ventilated dummy is used. The principle of trigger signal generation from multiple sensor signals is based on a self-developed algorithm that first evaluates the signal quality of each sensor based on adjustable parameters. Only the sensors selected as suitable are then used to calculate an averaged scaled signal, which is taken for trigger point detection. The best results were typically obtained when quality factures are set to a level where about half of the sensors are contributing to the trigger detection, leading to a trigger delay of about 80 ms relative to the pressure reference signal. It could also be shown that the algorithm can resume the trigger point detection within 2-3 seconds, after manually applying disturbances which could similarly occur in the clinical environment. The results show that the skin-friendly sensor patch provides suitable trigger signals for artificial respiration which are robust against drop out of single sensors, non-ideal sensor patch positioning on the thorax and mechanical irritations.
\end{abstract}

\section{Introduction}

Respiration monitoring is a widely used clinical method which is nowadays applied for all age classes, from preterm infants to elderly people. Since the respiratory rate is one of the four vital signs of the human body (besides body temperature, pulse rate and blood pressure), sensing its fluctuations is very important [1] because it can often directly indicate the medical condition of a patient and warn of various clinical deteriorations (dyspnea: short of breath, tachypnea: abnormal rapid breathing) or even of an impending total respiratory arrest (apnea) [2]. Patients diagnosed with any type of respiratory distress are often supported by applying artificial respiration, which can mainly fall in two categories: an invasive one, which is applied through a tracheal tube, and a more gentle and comfortable non-invasive method through a respiratory mask [3]. Although invasive respiration techniques have improved the medical treatment substantially, clinicians are moving away from usual intubation and search for non-invasive alternatives, especially for the ventilation of preterm infants, which are very prone to any kind of medical intervention [4]. Non-invasive respiration can for example increase survival rate by preventing "bronchopulmonary dysplasia" (BPD) [5]. One conventional noninvasive respiration method is the application of a "constant positive airway pressure" (CPAP), which prevents the lung from collapsing and hence supports own breathing of the patient [6]. Another non-invasive respiration method, which delivers more support to the infant than CPAP, is called intermittent positive pressure ventilation (NIPPV). This technique combines CPAP with intermitted pressure increases by generating peak pressures slightly higher than baseline CPAP. NIPPV alone already reduces work of breathing and synchronized NIPPV (SNIPPV) can reduce rate of reintubation [7]. It was also shown that synchronization of NIPPV can improve its effectiveness [8]. Generally, synchronization of ventilator inflations with the patient's own 
spontaneous breathing should lead to adequate gas exchange at lower airway pressures, reducing baro/volutrauma, air leaks and ventilation duration [9], which again can prevent BPD [10].

To be able to adapt airway pressures and flows to the patient's own breathing (synchronization), intelligent sensors are needed to detect the beginning and ending of the breathing cycle [11-15] . Existing synchronization systems use different approaches to detect the patient's own breathing; the electrical impedance tomography (EIT) [11] for example uses electrodes applied to the thorax which can measure its impedance changes during breathing, but it is very prone to motion artefacts and shows a relatively slow sensor response. Respiratory inductance plethysmography (RIP) [12] measures chest wall and abdominal movements via elastic bands and is a well-known and good functioning technique in respiration monitoring for adults. Unfortunately there are currently almost no readily available RIP systems for preterm infants [4]. Most commonly used systems for preterm infants are pneumatic abdominal capsules like the "Graseby capsule" (GC) [13] which show fast sensor response but are also very prone to motion artefacts. Since usually only one single capsule is used, it needs also a very accurate placement and runs the risk of relatively easy misplacement through body movements. Another system, called neurally adjusted ventilatory assist respiration (NAVA) [14] detects the breathing by measuring the electrical activity of the diaphragm; a relatively large and expensive electrode array is invasively applied to the diaphragm, limiting the usability for very small infants.

Foil-based microsystems that can be easily attached to bodies with non-planar shapes have received increasing attention in recent years. They are characterized by light weight and ultra-thin design that offers the flexibility required for a wide range of applications such as wearable electronics [16], structural health monitoring [17] and medical diagnostics [18]. However, flexibility alone is no longer sufficient when it comes to conformal application directly on the elastic human skin in order to achieve perfect shape adaption and wearing comfort. The concept of stretchable electronic systems [19] is much younger than flexible electronics [20]. It can be dated back to a paper published in 2001, which surveys the state-of-the-art and research issues that need to be resolved in order to make sensitive skin a reality [21]. Since then the recognized need for elasticity led to the development of numerous new materials and manufacturing technologies for stretchable electronics [22]. Own previous works include micro fabrication and characterization of a purely flexible sensor array [23] as well as conversion into a stretchable format with investigations on elasticity, shape adaptation and preliminary examination for trigger point recognition [24].

In contrast to the previously published works [23-25], this paper focuses on the realization of a stretchable sensor array by additional laser and embedding processes and specific algorithms developed for respiratory trigger signal generation based on 36 individual sensor signals. The use of such a foil-based stretchable sensor array for respiratory triggering could provide a promising alternative to existing synchronization systems [1115] and could overcome their typical drawbacks, especially in the application for preterm infants. The idea is to use the sensor as a non-invasive system by applying it in the transition between chest and abdomen to measure the typical oscillating deformations induced by breathing. It's ultra-thin and light-weight format with skin-like elastic properties allows perfect adaption to the body and thereby causes minimum stress to the infant, which makes it much more comfortable in comparison to other systems. The use of multiple sensors, evenly distributed on a 2D surface, could help to eliminate motion artefacts from the triggering signal and the drop out of one or even more sensors wouldn't cause a failure of the entire system. A multiple sensor structure could additionally minimize the risk of displacement and allow an easy placement by the operator. Since the lateral size of the sensor array can easily be adapted, it is applicable to any body size, even for very small preterm infants.

\section{Design of the stretchable sensor array}

A 6x6 sensor matrix foil as described earlier [23] is cut with fs-laser pulses to remove all parts of the polyimide foil which are not covered by sensors or electrical leads, resulting in small rigid sensor islands connected via slender meandering electrical leads. Afterwards, the already stretchable sensor array is placed on a $200 \mu \mathrm{m}$

Seite $\mathbf{2}$ von 14 
thick polydimethylsiloxane layer (PDMS) applied to a glass substrate. A further $200 \mu \mathrm{m}$ thick PDMS top layer is applied by spin coating to cover the sensors. After the PDMS has cured, the outer contour of the sensor is structured again using a laser. Finally, a stretchable sensor array with skin-like properties can be peeled from the carrier substrate. Figure 1 (a) shows the peeled off and stretched sensor array with 36 single sensor elements connected via meandering lines. Close up views of a single sensor island cut out be fs-laser pulses and of the slender meandering leads are given in Fig $\mathbf{1}(\mathbf{b}, \mathbf{c})$. Figure $\mathbf{1}(\mathbf{d})$ shows the orientation of each sensor element within the array structure, where four different orientations can be distinguished $\left(\mathrm{s}_{0}, \mathrm{~s}_{45}, \mathrm{~s}_{90}, \mathrm{~S}_{135}\right)$. Each sensor element consists of four strain gauges in a Wheatstone bridge configuration (see Figure 1 (f)). To achieve a higher sensitivity we placed two resistors ( $R 2, R 3)$ on one foil surface and two others (R1, R4) on the opposing foil surface as shown in Figure $\mathbf{1}$ (e). Foil bending accordingly leads to an elongation of resistors on one surface and to a compression of the resistors on the opposite surface, which results in a sensitivity increase of $170 \%$ compared to a "one-sided" sensor design (see [23] for more details).

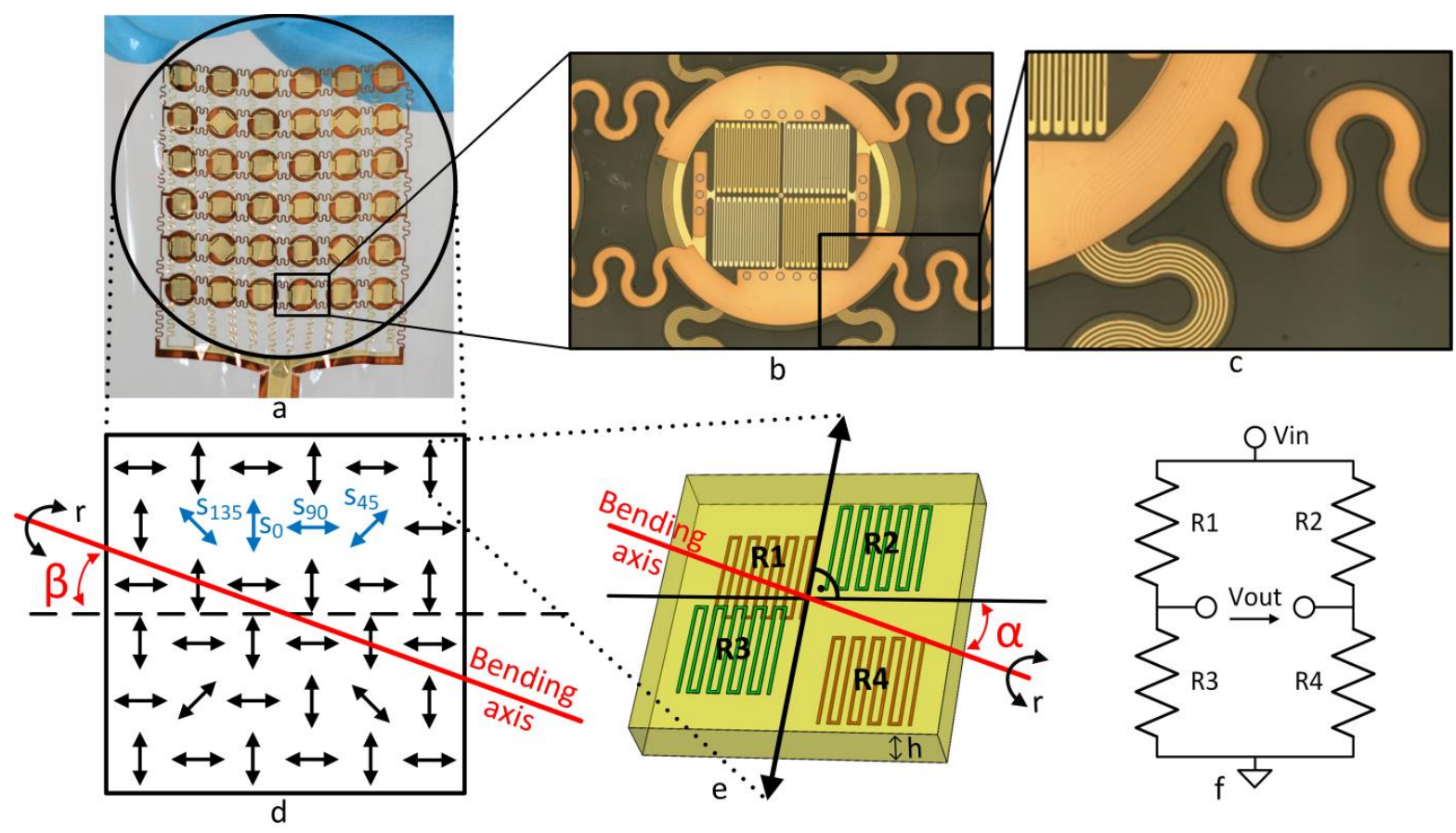

Figure 1: (a) 6x6 sensor array embedded in two layers of PDMS stretched by hand, (b) magnified view of one single sensor island structured with a fs-laser, (c) magnified view of slender meandering leads (d) indication of four different sensor orientations $\left(s_{0}, s_{45}, s_{90}, s_{135}\right)$ within the $6 \times 6$ sensor array and definition of bending angle $\beta$ for the entire sensor foil, (e) illustration of the double-sided sensor design and definition of bending angle $\alpha$ for one single sensor element, ( $f$ ) illustration of the appropriate connection to a full Wheatstone bridge

The bending induced output signal $V_{\text {out }}(\alpha, r)$ for our sensor is obtained as:

$$
V_{\text {out }}(\alpha, r)=V_{\text {in }} \cdot \frac{h}{r} \cdot\left[\frac{(1+q)}{2}+\frac{(1-q)}{2} \cdot \cos (2 \alpha)\right]
$$

$\alpha \quad=$ bending orientation angle

$r \quad=$ bending radius

$V_{\text {in }} \quad=$ Wheatstone bridge supply voltage (in our case $3.3 \mathrm{~V}$ )

$h \quad=$ interlayer thickness between top and bottom sensor layer (in our case $11.5 \mu \mathrm{m}$ )

$\mathrm{q}=$ = transversal sensitivity of a strain gauge (in our case 0.3 )

Factor $q$ is the ratio of transversal over longitudinal sensitivity which was experimentally obtained as $q=0.3$ in average by applying multiple measurements in longitudinal $\left(\alpha=0^{\circ}\right)$ and transversal direction $\left(\alpha=90^{\circ}\right)$ at bending radius of $r=7.5 \mathrm{~mm}$. To illustrate the advantage of a multisensory array with different sensor 
orientations we plotted the signal strength (according to Eq. 1 and normalized to $100 \%$ ) of each sensor orientation in dependence of the bending angle $\beta$ for the entire sensor array (see Figure 2 ).

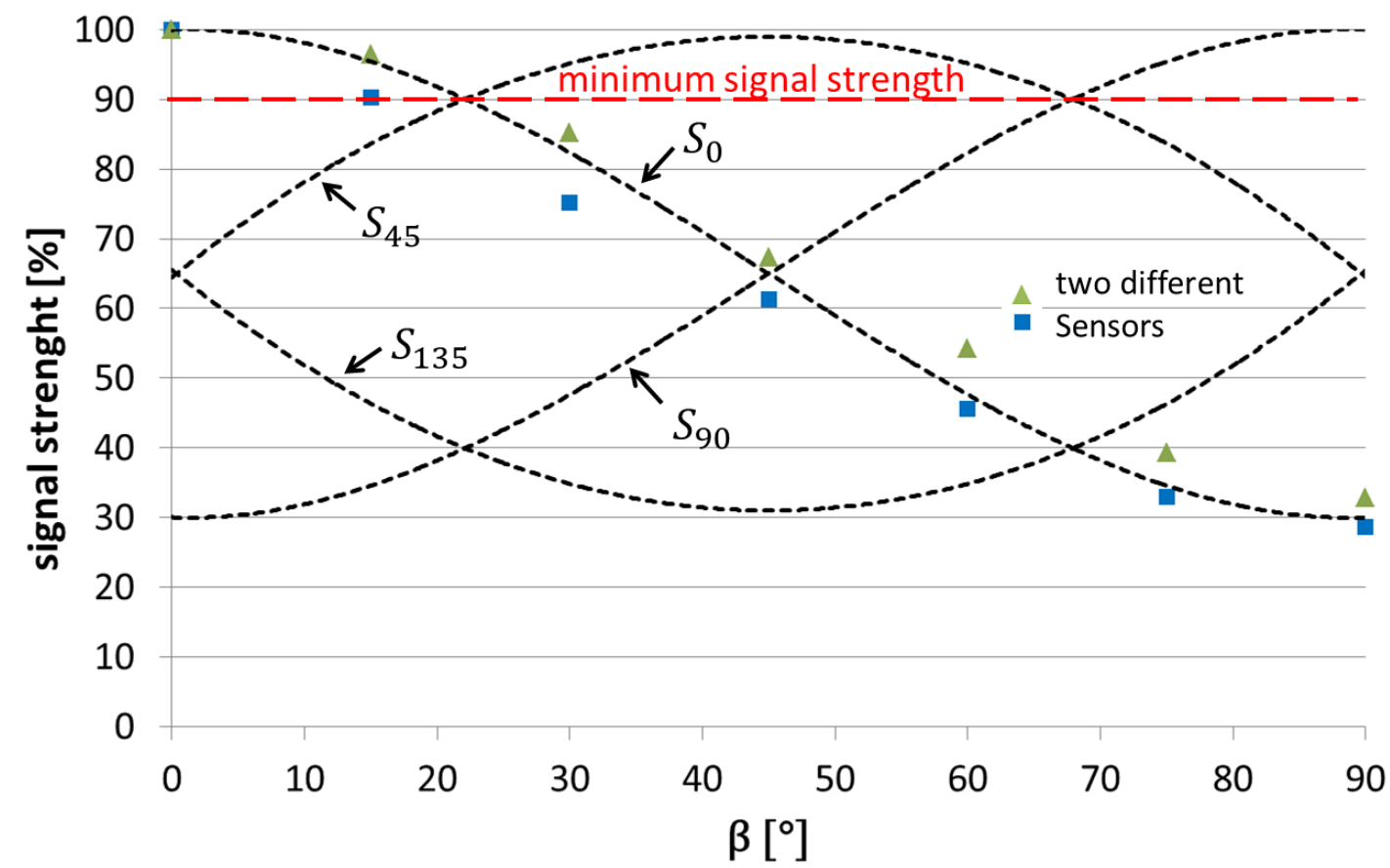

Figure 2: Signal strength (normalized to $100 \%$ ) for each of the four sensor orientations $\left(s_{0}, s_{45}, s_{90}, s_{135}\right)$ in dependence of the bending orientation angle $\beta$ for the entire sensor array. The dashed lines represent Eq. 1 , whereas the data points are experimentally obtained from two different sensors. The dashed red line at $\mathbf{9 0 \%}$ indicates the minimum strength which is provided by at least one sensor orientation at any angle $\beta$.

It can be recognized that for each bending angle $\beta$ sensors with minimally $90 \%$ of their longitudinal signal strength are available. The relationships between the bending angle $\beta$ for the entire sensor array and the bending angle $\alpha$ for each individual sensor are given as:

$$
\begin{array}{lll}
S_{0} & \rightarrow & \alpha=\beta \\
S_{45} & \rightarrow & \alpha=\beta+45^{\circ} \\
S_{90} & \rightarrow & \alpha=\beta+90^{\circ} \\
S_{135} & \rightarrow & \alpha=\beta+135^{\circ}
\end{array}
$$

The measurement points in Figure $\mathbf{2}$ are obtained from two different sensors and show good agreement with Eq. 1. For an intuitive understanding of sensor array operation, Figure $\mathbf{3}$ shows measured signal outputs of all 36 sensors (right) at bending in an S-shape (left). Both, the relationship between sensor orientation and signal strength as well as the change in signal sign due to the change in bending polarity can be very well recognized.
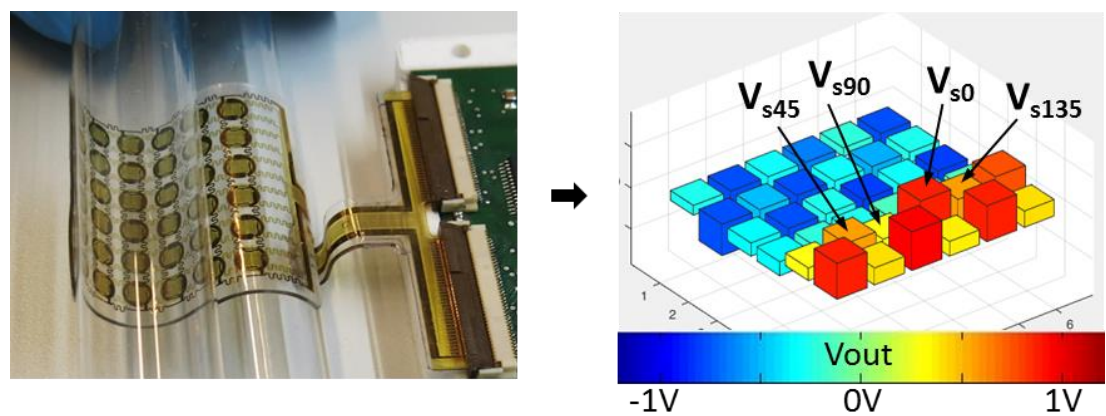

Figure 3: Illustration of sensor array operation: S-shape bending of sensor foil (left), graphic bar chart representation of all resulting 36 sensor output signals within the $6 \times 6$ sensor array 
Besides respiratory triggering, such a sensor array with bending-direction dependent sensor signals can also be used for recognition of its own current shape [25] and for providing additional diagnostic information as can be useful to detect diseases like pneumothorax [26].

\section{Experimental setup}

To simulate artificial respiration we built up an infant dummy as illustrated in Figure $\mathbf{4}$ (left). It can be externally ventilated by substituting the abdomen with an inflatable balloon connected to a respiration system (Figure 4 top right). To attach the sensor patch, it is placed on the balloon and wrapped with a stretchable foil. Due to the elasticity of all materials (balloon, sensor patch and stretchable wrap foil) and the natural adhesive properties of the PDMS, optimal conformity and slip resistance is achieved without the need for additional adhesive material.

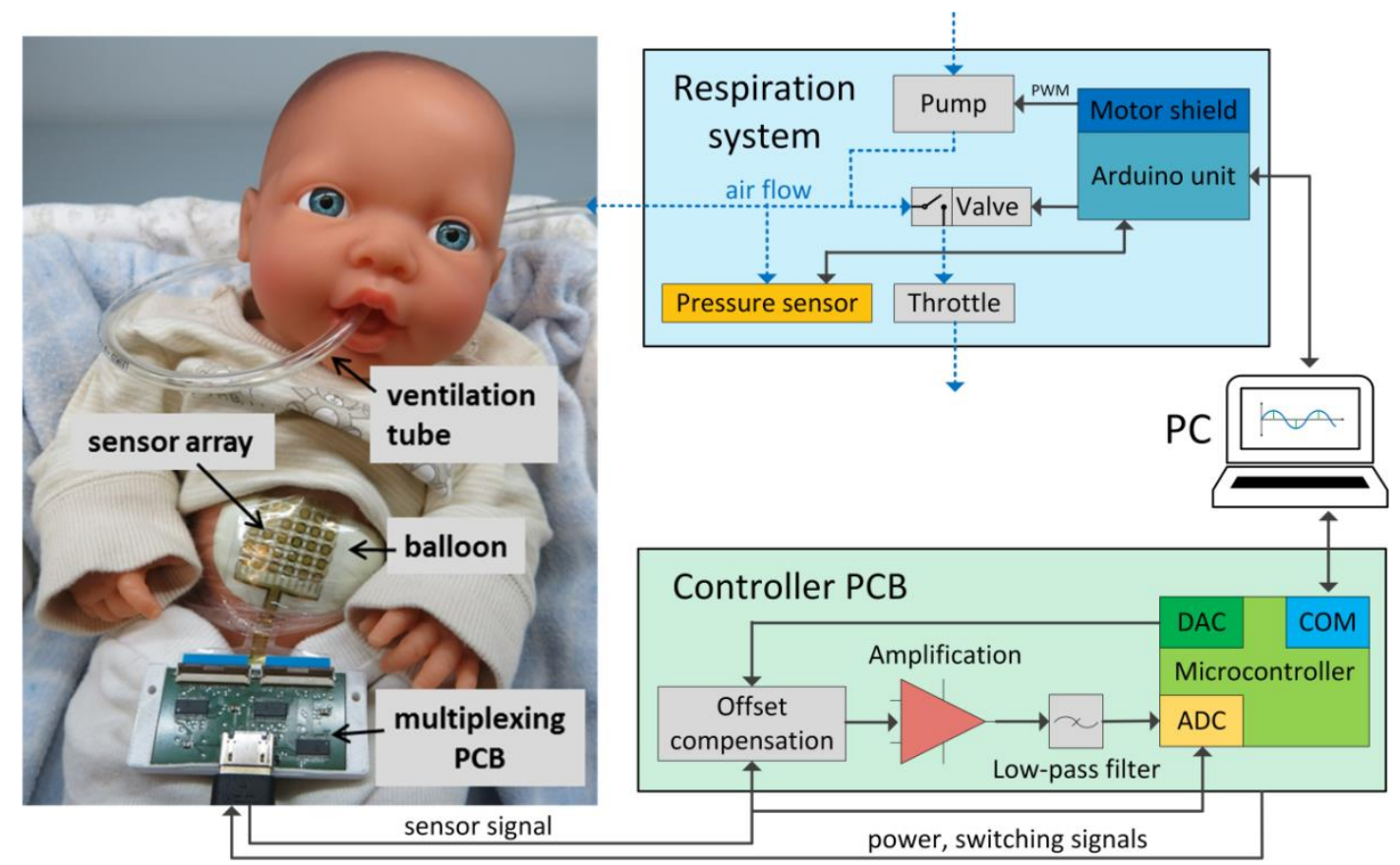

Figure 4: Illustration of measurement setup: photograph of respiration dummy with applied sensor array and multiplexing PCB (left), schematic illustration of the respiration system (top right) and controller PCB (bottom right) which are both controlled by a PC.

The air flow is generated using a conventional DC motor pump whose power is controlled by a pulse width modulation (PWM) signal from a driver unit (Arduino motor shield) enabling control of the inspiration flow rate. Since the pump is running continuously, a valve is used to control the air flow direction. With an open valve the air flows directly in to the dummy, otherwise through the valve and a throttle out of the system. The throttle is used to control the amount of outgoing air flow and hence allows a manual pre-adjustment of the expiration flow rate. The inspiration and expiration duration are digitally controlled by setting limits for the pressure, which is measured by an external sensor. Sensor signals are processed by home-built evaluation electronics, which consists of two main parts; a multiplexing $\mathrm{PCB}$ and a controller PCB, both connected via HDMI cable. The multiplexing PCB is directly connected to the sensor array via FFC-FPC connectors and converts the incoming 72 analog sensor signals in only 2 analog signals for the controller PCB. Before starting a measurement, the offsets of all sensors are determined. During measurement, the signal is offset compensated by a digital-to-analog (DAC) converter, then amplified and smoothed with a low-pass filter before it is measured with an analog-to-digital (ADC) converter. Finally, the signal is digitized by the microcontroller and sent via USB connection to the PC controlling the ventilation system for the data processing and visualization using MATLAB software. 


\section{Principle of trigger signal detection}

Figure 5 gives an overview of the complete trigger signal detection process in a flow chart representation.

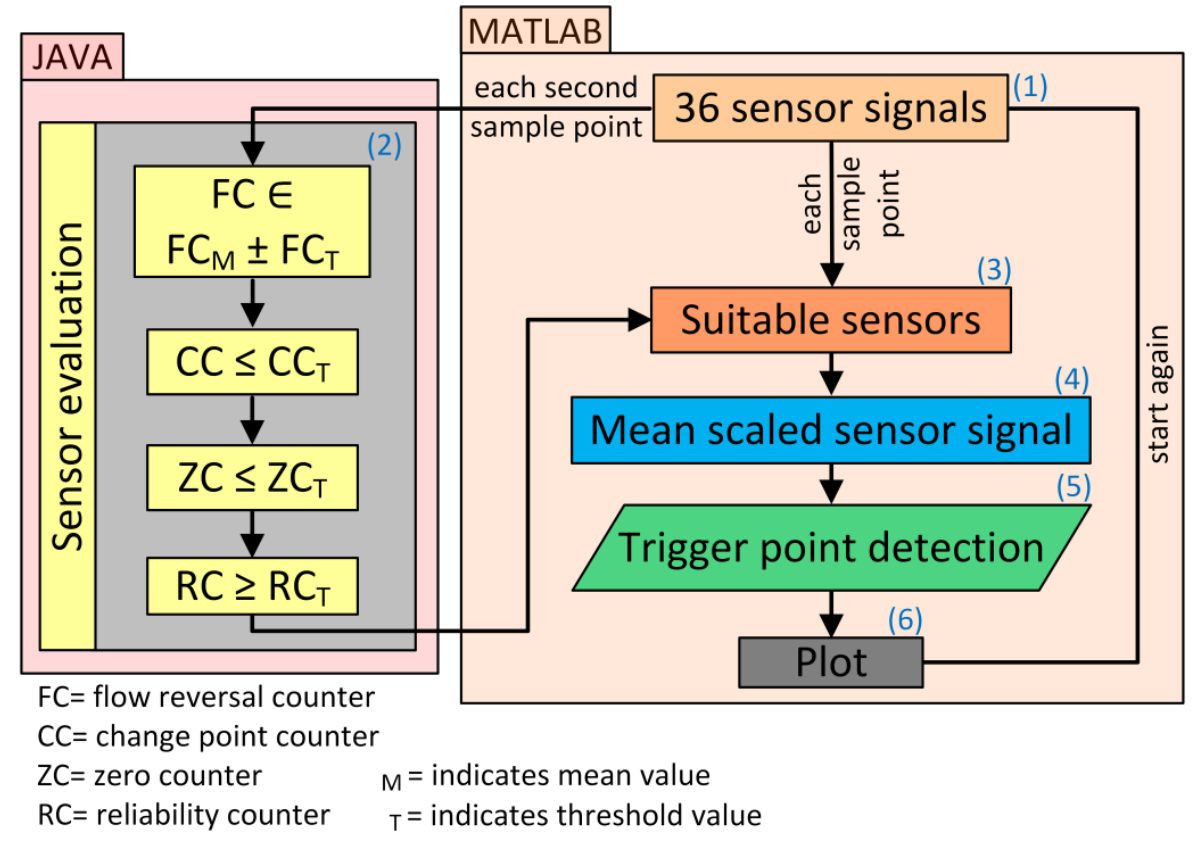

Figure 5: Flow chart representation of the trigger point detection process which is implemented in two separate software parts: JAVA software (left) and MATLAB software (right)

At the very beginning the signal quality of all 36 sensor signals (1) is evaluated by different criteria's (2) to select suitable sensors (3). Next, a mean of scaled sensor signal is calculated (4), which is used for the trigger point detection (5). If a trigger point is detected it is plotted (6) and the process starts again. The sensor evaluation process repeats every second sample point (approx. every $66 \mathrm{~ms}$ ) in a parallel running separate JAVA program whereas the trigger point detection within the core MATLAB program considers all sample points (repeats approx. every $33 \mathrm{~ms}$ ). The separation helps to increase speed performance, because otherwise the evaluation process can delay the trigger detection process. The core of the evaluation process is the reliable determination of signal slope polarity changes in a periodic breathing signal which result from the flow reversal between inspiration and expiration within a breathing cycle. Figure 6 illustrates the challenges of this process with a schematic showing an idealized signal and possible deviations occurring in real signals. 


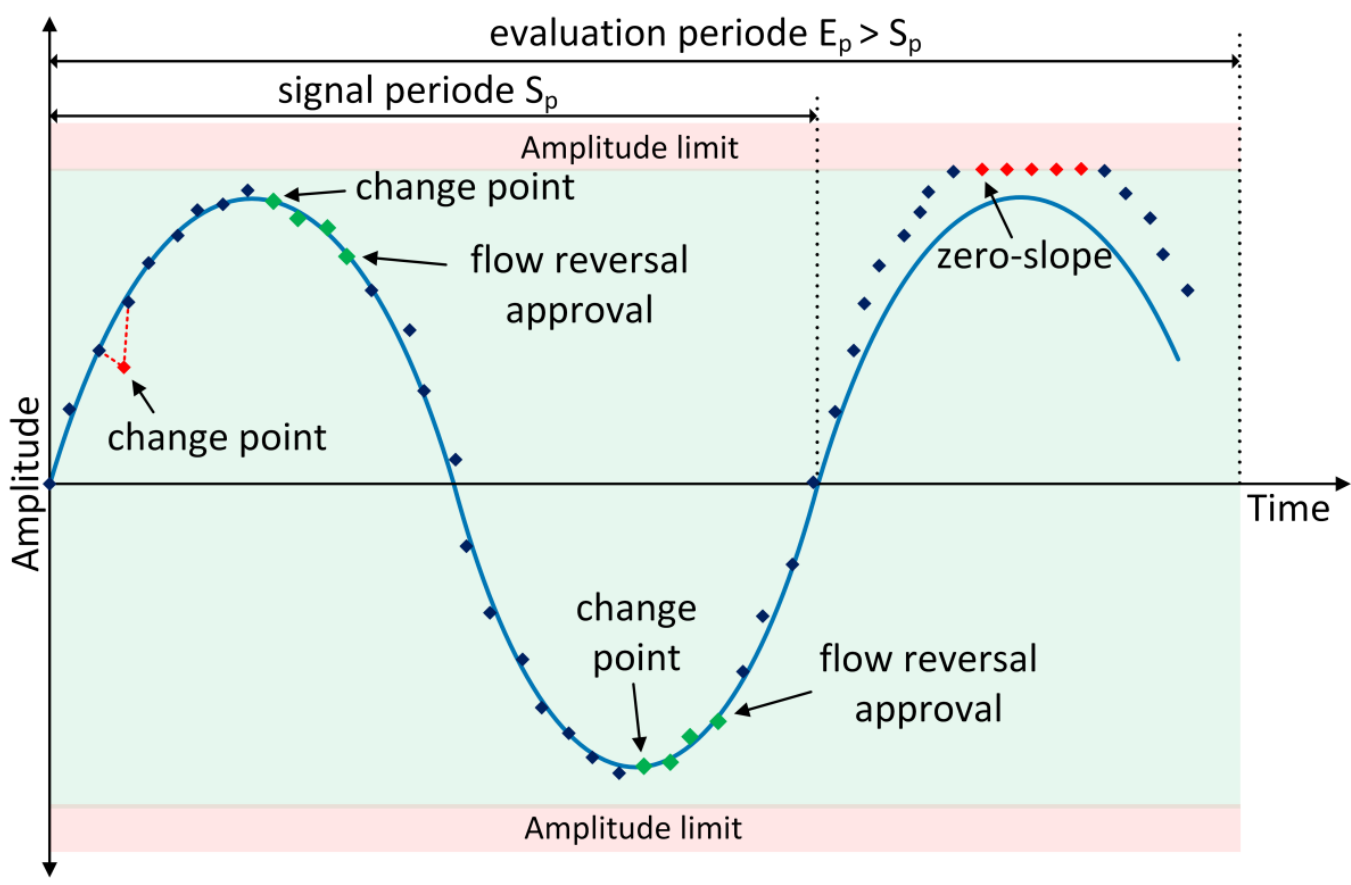

Figure 6: Schematic illustration of the challenges of flow reversal determination in a typical course of a realistic signal (dots) which is compared to an idealized periodic signal (continuous curve).

The evaluation period $E_{p}$ has to exceed the signal period $S_{p}$, which ensures that at least one complete signal period is evaluated. This also means that at least one evaluation period must be recorded before the first trigger point can be detected. To obtain a signal which is more reliable and closer to an idealized signal than one of the 36 individual sensor signals, in a first step not all of the individual sensor signals are considered. The selection of sensors is based on four different criteria, which are indicated in Figure $\mathbf{6}$ and will be described in the following more in detail.

The Flow reversal Counter $(F C)$ counts the number of signal polarity changes resulting from the periodic flow reversal between inspiration and expiration. We consider only three types of signal slopes: positive (+), negative (-) and zero (0). A flow reversal point is only counted if the signal slope polarity changes and remains for at least four consecutive sample points as illustrated in Figure 7 (left). The Change point Counter (CC), on the other hand, counts the number of signal slope polarity changes that do not lead to a countable flow reversal point as illustrated with an example in Figure 7 (right).

flow reversal $(\mathrm{FC}+1)$ no flow reversal $(\mathrm{CC}+1)$
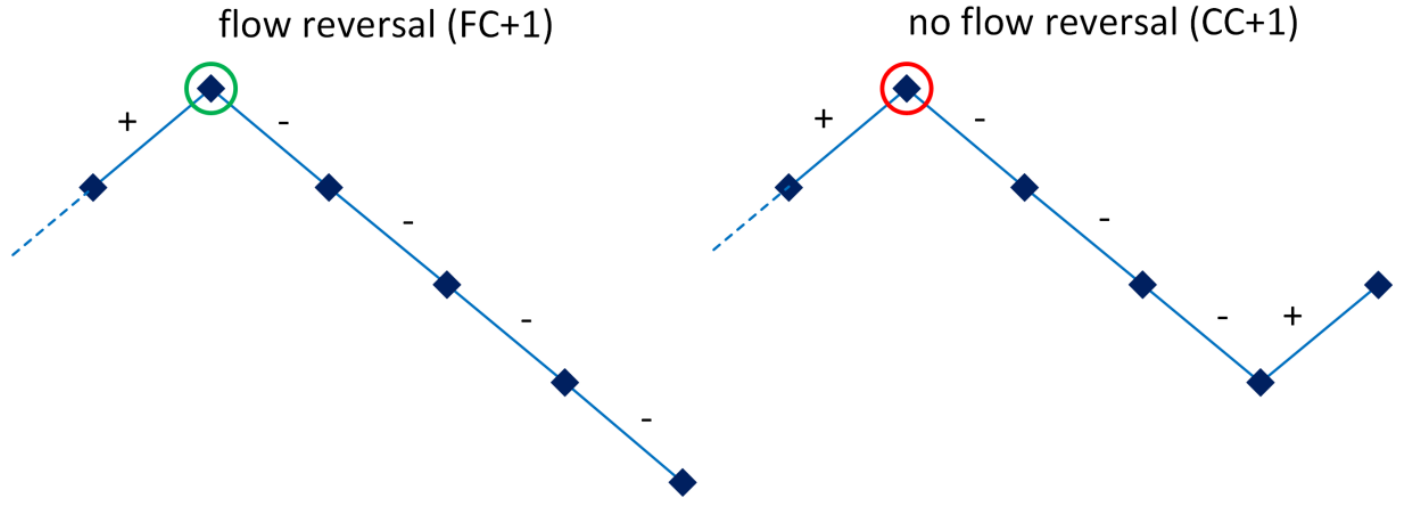

Figure 7: Schematic illustration of possible signal paths to detect a flow reversal (left) or a simple change point (right)

The Zero slope Counter (ZC) counts the amount of consecutive zero slopes. It is thereby able to identify sensors that do not respond at all and sensors with signals that exceed the dynamic range of evaluation electronics (+/$1.2 \mathrm{~V})$ as illustrated in Figure 6. The Reliability Counter $(R C)$ considers the last 10 evaluation results from the 
first three parameter (FC, CC, ZC) and counts how often each sensor was considered to be suitable for trigger point detection. The decision whether an individual sensor will be considered or not is based on adjustable threshold values $\left(F C_{T}, C C_{T}, Z C_{T}, R C_{T}\right.$ ) which are compared with the counter values ( $F C, C C, Z C, R C$ ), whereas for the flow reversal counter a mean value $\mathrm{FC}_{\mathrm{M}}$ of all sensors is also determined first in order to compare an interval of $\mathrm{FC}_{\mathrm{M}} \pm \mathrm{FC}_{\mathrm{T}}$ (see Figure 5).

After sensor evaluation, we apply scaling of each suitable sensor signal to obtain values from 0 to 0.5 , independent from the periodic signal phase. Due to their position some of the sensors can provide no signals and some can also provide periodic signals in counter phase which are acceptable since flow reversal points do not depend on polarity. To obtain comparable signals which are independent from the periodic signal phase, maximum $\left(\mathrm{V}_{\max }\right)$, minimum $\left(\mathrm{V}_{\min }\right)$, and mean values $\left(\mathrm{V}_{\text {mean }}\right)$ for each suitable sensor are determined within the evaluation period $E_{p}$ and a scaled signal $\left(\mathrm{V}_{\text {scaled }}\right)$ is calculated as:

$$
\mathrm{V}_{\text {scaled }}=\left|\frac{\mathrm{V}_{\text {out }}-\mathrm{V}_{\text {mean }}}{\mathrm{V}_{\max }-\mathrm{V}_{\min }}\right|
$$

Another benefit of scaled signals is their stability against any fluctuation in signal offset and amplitude. Since expiration takes about twice as long as the inspiration for a typical breathing cycle, a distinction between inspiration and expiration can still be obtained by considering the time periods between trigger points. In the final step an average of all selected and scaled sensor signals is calculated. Figure 8 illustrates the trigger point detection by identifying the point of flow reversal.

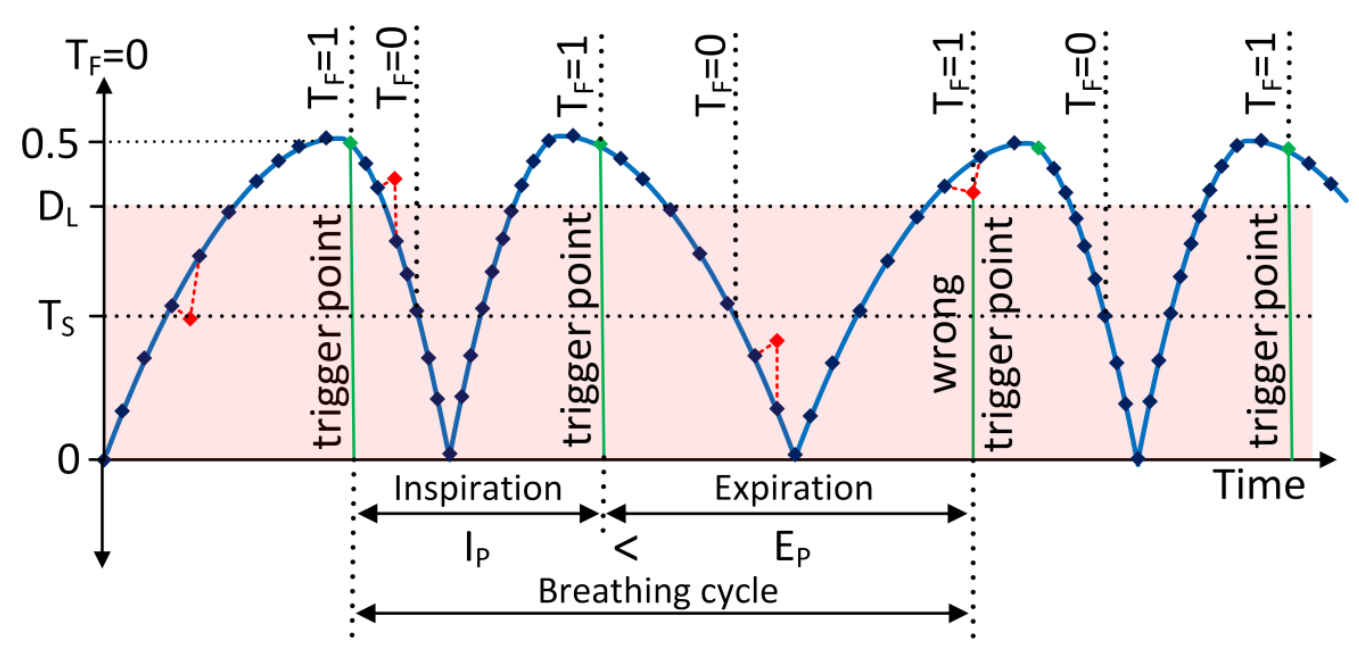

Figure 8: Schematic illustration of the trigger point detection: $D_{L}=$ detection limit, $T_{S}=$ trigger setback, $T_{F}=$ trigger flag

To avoid false trigger point detection we defined a threshold parameter $\mathrm{D}_{\mathrm{L}}$ which has to be surpassed by the mean signal to permit trigger point detection (red area indicates the signal range excluded for trigger point detection). Further, the trigger flag parameter $\mathrm{T}_{\mathrm{F}}$ ensures that only one trigger point can be detected within one half of a signal period. $\mathrm{T}_{\mathrm{F}}$ is set to one after a trigger point is detected. It remains at one until the signal reaches an adjustable trigger setback value $\mathrm{T}_{\mathrm{S}}$ at which time it is set to zero again. $\mathrm{A}$ trigger point can only be detected if $\mathrm{T}_{\mathrm{F}}=0$.

\section{Proof of concept for the trigger point detection}

The usefulness of multi-sensor evaluation is illustrated in Figure 9, showing arbitrarily selected ten raw signals of individual sensors together with the mean scaled sensor signal, which is automatically calculated by the evaluation algorithm according to Eq.3. 


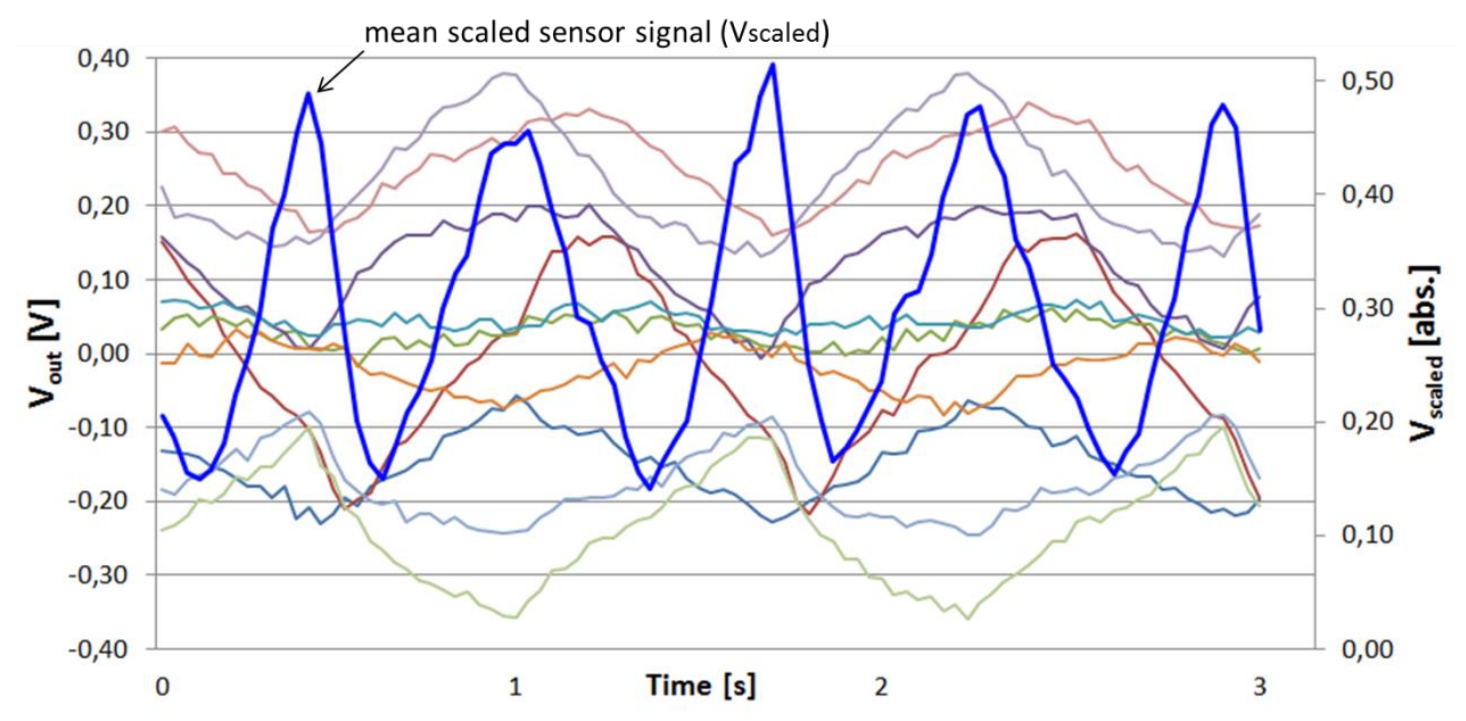

Figure 9: Computed mean scaled sensor signal (bold blue curve which gives $\mathrm{V}_{\text {scaled }}$ averaged over all sensors that were selected by the evaluation algorithm) together with ten raw sensor signals $\mathrm{V}_{\text {out }}$ (other curves). The ten raw sensor signals were arbitrarily selected to show possible differences in signal characteristics and do not resemble the group of signals selected to calculate the mean scaled sensor signal.

The raw sensor signals differ considerably in phase, amplitude and offset on the one hand and on the other hand they show frequent changes of the signal slope polarity, which would complicate the detection of trigger points. The mean scaled sensor signal, in contrast, shows uniform amplitude with almost no change in signal slope polarity between the expected extreme values. However, the amplitude of the mean scaled sensor signal does not oscillate between 0 and 0.5 , but rather shows lower amplitude, which is a result of small phase shift between the raw sensor signals.

To illustrate functionality of the trigger point detection algorithm, we recorded and analyzed the same measurement sequence with three different sets of threshold parameters as illustrated in Figure 10. The breathing signal was generated with $\approx 0.8 \mathrm{~Hz}$. The evaluation period $\mathrm{E}_{\mathrm{p}}$ has been set to 60 sampling points, which corresponds to $2 \mathrm{~s}$ at a sampling rate of $30 \mathrm{~Hz}$. The $\mathrm{CC}_{\mathrm{T}}$ is expressed as a percentage of the 60 sampling points, while the other threshold parameters are given in absolute values. 


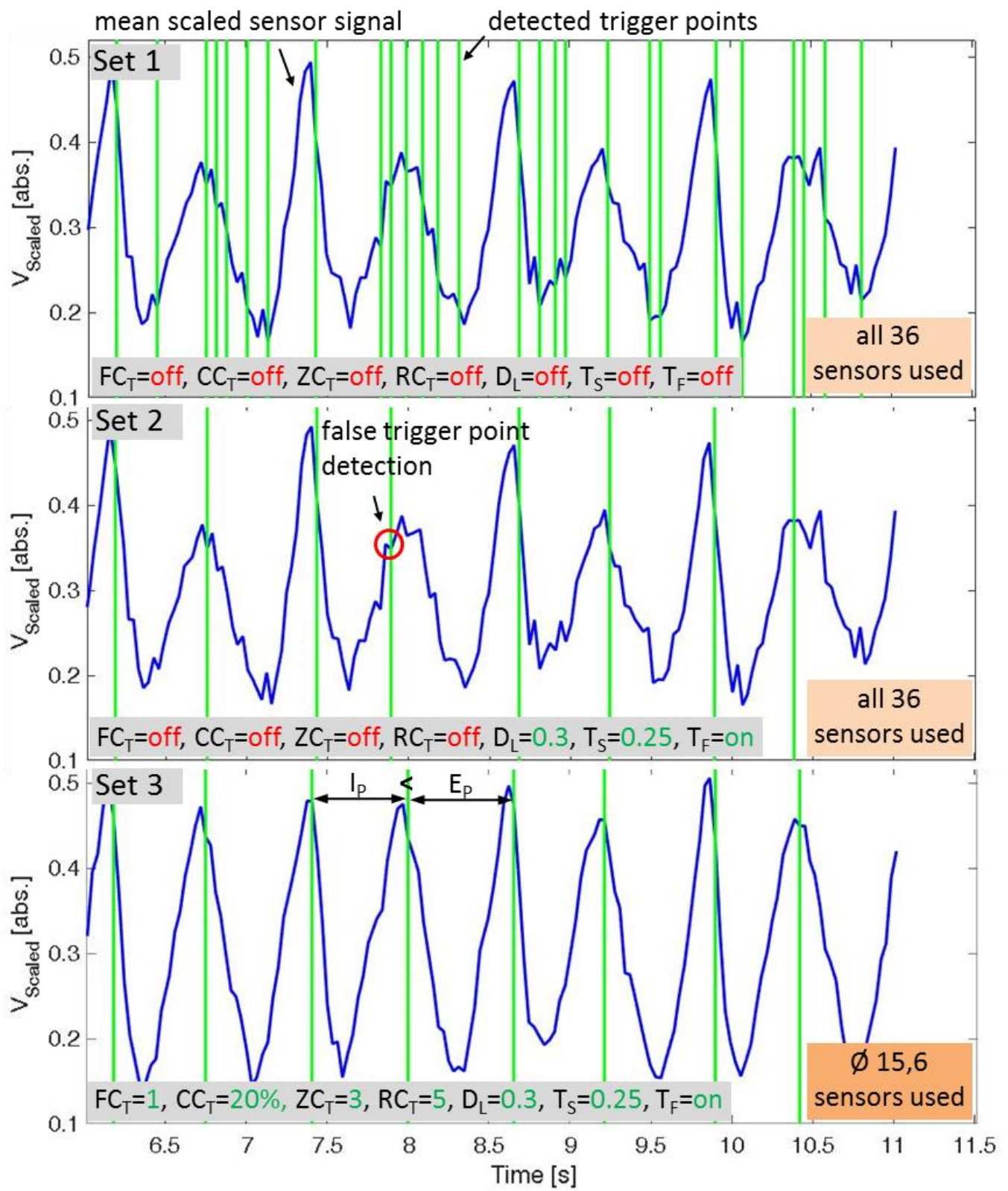

Figure 10: Diagrams, each showing a measurement section of $5 \mathrm{~s}$ from a trigger point detection sequence analyzed with three different sets of parameters: upper diagram: all parameters off, middle diagram: only the parameters detection limit $D_{L}$, trigger setback $T_{S}$ and trigger flag $T_{F}$ are on, bottom diagram: all parameters on, in addition the inspiration period $I_{p}$ and expiration period $E_{p}$ are indicated

Without sensor evaluation ( $\mathrm{FC}_{\mathrm{T}}=$ off, $\mathrm{CC}_{\mathrm{T}}=$ off, $\mathrm{ZC}_{\mathrm{T}}=$ off, $\mathrm{RC}_{\mathrm{T}}=$ off) all 36 sensors are used to calculate the mean scaled sensor signal in sequence $1 \& 2$. A much more irregular signal is observed when compared to sequence 3 , where in average only the best 15.83 sensors are used. Although the sensor evaluation results in a significantly better signal, the use of only the three parameters detection limit $D_{L}$, trigger setback $T_{S}$ and trigger flag $T_{F}$ already shows a very good trigger point detection. In sequence 1 each change in signal slope polarity would lead to a trigger signal whereas in sequence 2 the number of detected trigger points is reduced and consistent with the pressure excitation. Further, all trigger points are correctly detected, except for only one that is detected at a false position, leading to a "negative trigger delay" (see Figure 11 and Table 1). However, the best results with regard to signal quality and correctly detected trigger points shows sequence 3 .

In order to evaluate our algorithm, first the trigger point delay $T_{d}$ is introduced in Figure 11, representing the delay between extreme values (red arrows) in the pressure reference signal (red curve) and the detected 
trigger points (green vertical lines) obtained from the calculated scaled mean sensor signal (blue curve). The reference signal is provided by the pressure sensor from the respiration system as shown in Figure 4.

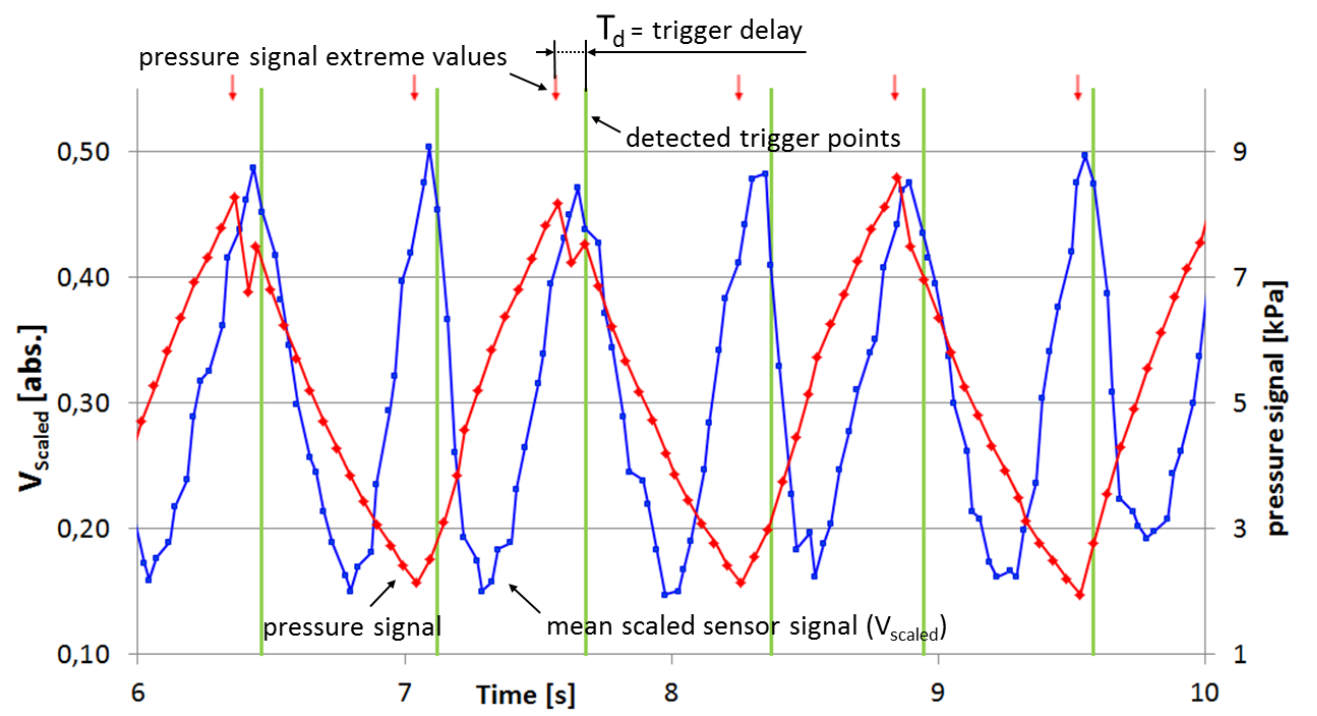

Figure 11: Pressure reference signal (red curve) and the detected trigger points (green vertical lines) from a scaled mean sensor signal (blue curve) with the trigger delay $T_{d}$.

Table 1 shows results of trigger point detection at varying sensor evaluation parameters $\mathrm{FC}_{\mathrm{T}}, \mathrm{CC}_{\mathrm{T}}, \mathrm{ZC}_{\mathrm{T}}$. The mean trigger delay $T_{d}$ (column 3 ) and the trigger delay standard deviation $\boldsymbol{\sigma}$ (column 4) are given. The number of suitable sensors determined by the alghorithm is shown in column 5 . The number of detected trigger points from a total of 80 flow reversals is shown in column 6 , where trigger points with premature occurrence in regard to the pressure extreme values are counted as negative and with delayed occurance as positive. Extreme pressure values that do not correlate with a trigger point detection are counted as not-detected. The green highlighted row represent obtained optimal results for this specific measurement.

Table 1: Results of trigger point detection by varying the sensor evaluation parameter $C_{T}\left(F_{T}=1, R C_{T}=5, Z C_{T}=3\right)$

\begin{tabular}{|c|c|c|c|c|c|}
\hline Nr. & $\begin{array}{c}\mathrm{CC}_{\mathrm{T}} / \mathrm{FC}_{\mathrm{T}} / \mathrm{RC}_{\mathrm{T}} / \mathrm{ZC}_{\mathrm{T}} \\
\text { [\%/abs./abs./abs.] }\end{array}$ & $\begin{array}{l}\varnothing \mathrm{T}_{\mathrm{d}} \\
{[\mathrm{ms}]}\end{array}$ & $\begin{array}{c}\varnothing \sigma \\
{[\mathrm{ms}]}\end{array}$ & $\begin{array}{c}\varnothing \\
\text { Amount } \\
\text { sensors }\end{array}$ & $\begin{array}{c}\text { Detected trigger points } \\
\text { from a total of } 80 \\
\text { (positive/negative/not-detected) }\end{array}$ \\
\hline 1 & $0 / 1 / 5 / 3$ & 148,7 & 294,2 & 1,0 & $61 / 4 / 15$ \\
\hline 2 & $5 / 1 / 5 / 3$ & 93,8 & 35,6 & 4,4 & $79 / 1 / 0$ \\
\hline 3 & $10 / 1 / 5 / 3$ & 81,7 & 21,9 & 8,0 & $79 / 1 / 0$ \\
\hline 4 & $20 / 1 / 5 / 3$ & 77,1 & 21,8 & 15,6 & $79 / 1 / 0$ \\
\hline 5 & $30 / 1 / 5 / 3$ & 76,3 & 27,7 & 23,1 & $79 / 1 / 0$ \\
\hline 6 & $40 / 1 / 5 / 3$ & 78,5 & 45,6 & 27,3 & $75 / 5 / 0$ \\
\hline 7 & $100 / 1 / 5 / 3$ & 76,6 & 34,1 & 28,1 & $76 / 4 / 0$ \\
\hline 8 & $100 / 3 / 5 / 3$ & 74,7 & 44,2 & 35 & $70 / 10 / 0$ \\
\hline 9 & $100 / 3 / 5 / 5$ & 75,7 & 45,2 & 36 & $69 / 11 / 0$ \\
\hline
\end{tabular}

In general, we observed that the change point counter CC setting has the prominent influence on sensor filtering. The best results are obtained with $\mathrm{CC}_{\mathrm{T}}$ in the range of $20 \%$, leading to approx. 15.6 selected sensors, which gives us a trigger delay of approx. $77 \mathrm{~ms}$ and a standard deviation of approx. $22 \mathrm{~ms}$. For lower values of $\mathrm{CC}_{\mathrm{T}}$ the amount of used sensors decreases rapidly, which results in higher trigger delays with high standard deviations. At $C C_{T}=0$, sections in the course of the signal without any suitable sensor were observed, so that no trigger points could be detected. For higher values of $\mathrm{CC}_{\mathrm{T}}$ the amount of used sensors increases, which results in a decrease of signal quality as illustrated in Figure 10. This again leads to more trigger points detected too 
early. At $\mathrm{CC}_{\mathrm{T}}=100 \%$ a saturation of approx. 28 suitable sensors is reached, the remaining eight sensors are filtered by the further parameters. This is initially demonstrated in row 8 by increasing the $\mathrm{FC}_{\mathrm{T}}$ to 3 , resulting in 35 suitable sensors and then increasing the $\mathrm{ZC}_{\mathrm{T}}$ to 3 , resulting in all 36 suitable sensors. In general, after the $\mathrm{CC}$ was set for a specific measurement, the remaining parameters have only a minor effect. The RC predominantly rejects sensors for measurements with high signal fluctuations whereas the complete switching off $(R C=0)$ always leads to the worst results. This analysis is carried out under well-defined conditions, which are provided by the experimental set-up for simulating breathing. In a true medical application significantly higher signal fluctuations can occur and the sensor foil itself may show malfunctions due to the drop out of some sensors or when reaching the amplitude limit. In this case, the use of a multiple sensor array with all introduced parameters can be decisive.

Figure 12 shows the behavior of the sensor array in case of external disturbances, which are caused by simply touching the sensor array during the measurement. It is visible that the trigger point detection resumes after approx. 2-3 seconds, which corresponds to the duration of one evaluation period $E_{p}$.

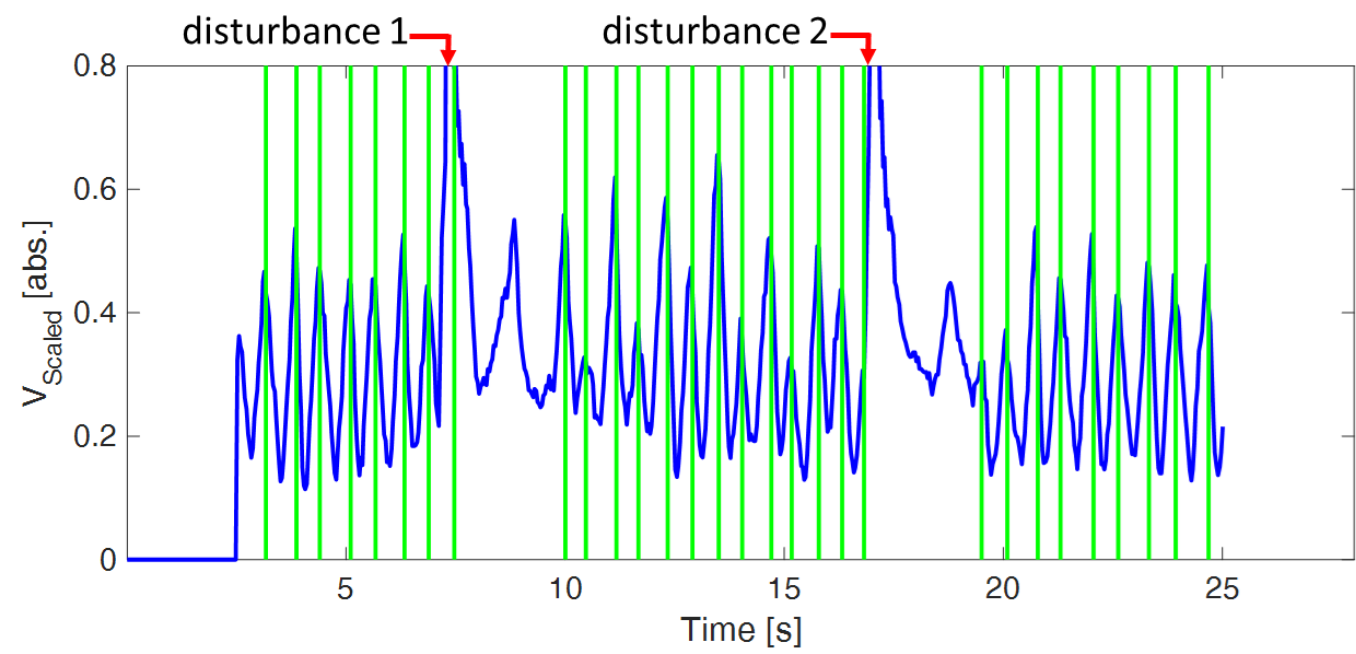

Figure 12: The course of calculated scaled mean sensor signal (blue curve) with temporary irritations (disturbances 1 and 2) which were manually induced. The trigger point detection resumes after 2-3 seconds.

\section{Summary and outlook}

A novel, stretchable sensor array was realized by converting a flexible sensor foil into a stretchable format by fs-laser structuring and subsequent embedding in PDMS. The suitability of this stretchable sensor foil for trigger signal detection in artificial respiration was investigated with a pressure driven set-up, simulating thorax deformations during breathing. A pressure sensor is used to provide a reference signal for our sensor evaluation. The detection of points that could be used for triggering an artificial respiration is based on an algorithm that first evaluates the signal quality of all 36 sensors using various adjustable threshold parameters. Sensors not suitable for the trigger point detection can be ignored. The remaining signals are then used to generate an averaged scaled signal, which is used for the trigger point detection. The best results were obtained when the algorithm identified about 15 of 36 sensors as suitable, which lead to a minimum trigger delay of down to approx. $77 \mathrm{~ms}$ and a standard deviation of approx. $17 \mathrm{~ms}$, compared to the pressure reference signal. It could also be shown that the algorithm can resume the trigger point detection within 2-3 seconds, after manually applying external disturbances. However, the successfully demonstrated feasibility of using the sensor array to detect the breathing cycle and provide trigger points for artificial respiration has been obtained with optimized parameters for a particular measurement and can deviate considerably for other measurements. The determination of trigger points on a real patient should be carried out in a following step. In addition, more general concepts for multi-sensor evaluation based on advanced algorithms such as Kalman filtering should be investigated. 


\section{Acknowledgements}

The authors are grateful for the funding provided by the German Ministry of Economics and Technology (BMWi) under the ZIM program (Zentrales Innovationsprogramm Mittelstand) with the funding reference KF3085603TS4

\section{References}

[1] F.Q. Al-Khalidi, R. Saatchi, D. Burke, H. Elphick, S. Tan, Respiration rate monitoring methods: A review, Pediatric pulmonology 46 (2011) 523-529.

[2] D. Hess, N.R. Maclntyre, W.F. Galvin, S.C. Mishoe, Respiratory care: Principles and practice, Jones \& Bartlett Learning, Burlington MA, 2016.

[3] L. Brochard, Mechanical ventilation: Invasive versus noninvasive, European Respiratory Journal 22 (2003) 31s-37s.

[4] L.S. Owen, B.J. Manley, P.G. Davis, L.W. Doyle, The evolution of modern respiratory care for preterm infants, The Lancet 389 (2017) 1649-1659.

[5] H.S. Fischer, C. Buhrer, Avoiding Endotracheal Ventilation to Prevent Bronchopulmonary Dysplasia: A Meta-analysis, PEDIATRICS 132 (2013) e1351-e1360.

[6] IEEE, INTELLIGENT CPAP - Sleep Monitoring, IEE Colloquium on.

[7] C.T. Roberts, P.G. Davis, L.S. Owen, Neonatal non-invasive respiratory support: Synchronised NIPPV, non-synchronised NIPPV or bi-level CPAP: what is the evidence in 2013?, Neonatology 104 (2013) 203-209.

[8] P.G. Davis, B. Lemyre, A.G. de Paoli, Nasal intermittent positive pressure ventilation (NIPPV) versus nasal continuous positive airway pressure (NCPAP) for preterm neonates after extubation, The Cochrane database of systematic reviews (2001) CD003212.

[9] A. Greenough, G. Dimitriou, M. Prendergast, A.D. Milner, Synchronized mechanical ventilation for respiratory support in newborn infants, The Cochrane database of systematic reviews (2008) CD000456.

[10] G. Bernstein, F.L. Mannino, G.P. Heldt, J.D. Callahan, D.H. Bull, A. Sola, R.L. Ariagno, G.L. Hoffman, I.D. Frantz, B.I. Troche, J.L. Roberts, Dela Cruz, Teddy V., E. Costa, Randomized multicenter trial comparing synchronized and conventional intermittent mandatory ventilation in neonates, The Journal of Pediatrics 128 (1996) 453-463.

[11] F.S. Rossi, A.C. Yagui, L.B. Haddad, A.A. Deutsch, C.M. Rebello, Electrical impedance tomography to evaluate air distribution prior to extubation in very-low-birth-weight infants: a feasibility study, Clinics 68 (2013) 345-350.

[12] J.A. Adams, I.A. Zabaleta, D. Stroh, P. Johnson, M.A. Sackner, Tidal volume measurements in newborns using respiratory inductive plethysmography, The American review of respiratory disease 148 (1993) 585-588.

[13] D.J. Stern, M.D. Weisner, S.E. Courtney, Synchronized neonatal non-invasive ventilation-a pilot study: the graseby capsule with bi-level NCPAP, Pediatric pulmonology 49 (2014) 659-664.

[14] H. Stein, K. Firestone, Application of neurally adjusted ventilatory assist in neonates, Seminars in fetal \& neonatal medicine 19 (2014) 60-69.

[15] M.F. Hird, A. Greenough, Comparison of triggering systems for neonatal patient triggered ventilation, Archives of Disease in Childhood 66 (1991) 426-428.

[16] M. Stoppa, A. Chiolerio, Wearable electronics and smart textiles: A critical review, Sensors 14 (2014) 11957-11992. 
[17] B. Glisic, Y. Yao, S.-T.E. Tung, S. Wagner, J.C. Sturm, N. Verma, Strain Sensing Sheets for Structural Health Monitoring Based on Large-Area Electronics and Integrated Circuits, Proc. IEEE 104 (2016) 1513-1528.

[18] J. Smith, Flexible Electronics and Display Technology for Medical, Biological, and Life Science Applications, Arizona State University (Dissertation 2014).

[19] T. Someya, Stretchable electronics, Wiley-VCH, Weinheim, 2013.

[20] W.S. Wong, A. Salleo, Flexible electronics: Materials and applications, Springer, New York, 2009.

[21] Vladimir J. Lumelsky, Michael S. Shur, Sigurd Wagner, Sensitive skin - Sensors Journal, IEEE (2001).

[22] T. Someya, Stretchable electronics, Wiley-VCH, Weinheim, 2013.

[23] E. Koch, A. Dietzel, Skin attachable flexible sensor array for respiratory monitoring, Sensors and Actuators A: Physical 250 (2016) 138-144.

[24] E. Koch, A. Dietzel, Stretchable sensor array for respiratory monitoring, in: 2017 19th International Conference on Solid-State Sensors, Actuators and Microsystems (TRANSDUCERS), Kaohsiung, Taiwan, IEEE, 2017, pp. 2227-2230.

[25] E. Koch, A. Dietzel, Surface reconstruction by means of a flexible sensor array, Sensors and Actuators A: Physical (2017).

[26] D. Waisman, A. Landesberg, S. Kohn, A. Faingersh, I.C. Klotzman, A. Gover, I. Kessel, A. Rotschild, Chest dynamics asymmetry facilitates earlier detection of pneumothorax, J Perinatol 36 (2016) 157-159. 